\title{
EFEITO DO MEIO E DA IDADE DA VACA SOBRE O GANHO DE PESO NA FASE DE CRIA, RECRIA E OS DIAS PARA SE OBTER 160 KG EM ANIMAIS DA RAÇA GUZERÁ (Environmental effects and age of dam on pre- and post-weaning daily gain, and on number of days to gain $160 \mathrm{~kg}$ from birth to weaning on guzerath breed cattle)
}

\author{
GEMIN, E. ${ }^{1}$; SOUZA, J.C. ${ }^{2}$; SILVA, L.O.C. ${ }^{3}$ MALHADO, C.H.M. ${ }^{4}$; FERRAZ FILHO, P.B. ${ }^{5}$ \\ ${ }^{1}$ Acadêmica de graduação - UFPR, PR; \\ 2Departamento de Zootecnia - UFPR, PR - jcs@ufpr.br. Rua dos Funcionários 1540. Curitiba, PR. \\ CEP 80035-050; \\ ${ }^{3}$ Pesquisador CNPGC/Embrapa, MS; \\ ${ }^{4}$ Pós Graduando IBB Genética - Un; \\ ${ }^{5}$ Prof. Adjunto, UFMS, MS.
}

RESUMO - O objetivo deste trabalho foi avaliar a influência dos efeitos de meio e da idade da vaca sobre os ganhos de peso do nascimento ao desmame (GPD), período pós-desmame (GPS) e sobre o número de dias para se obter $160 \mathrm{~kg}$ (D160). O rebanho avaliado continha 1.747 animais, sendo os dados analisados pelo método dos quadrados mínimos utilizando-se um modelo estatístico contendo os efeitos fixos de mês, ano e sexo do bezerro, o efeito aleatório de touros na fazenda, e como covariável a idade da vaca ao parto. As médias ajustadas para ganho de peso pré e pós-desmame, e para dias para a obtenção $160 \mathrm{~kg}$ foram 0,604 $\pm 0,01 \mathrm{~kg}$; $0,399 \pm 0,01 \mathrm{~kg}$; em $285 \pm 5,3$ dias, respectivamente. Os machos foram superiores às fêmeas relativo ao GPD $=6,0 \%$; D160 $=5,8 \%$, GPS $=20,1 \%$. Quanto ao mês, as maiores médias de ganho de peso no pré-desmame recaiu nos animais nascidos no mês de agosto. Com relação aos dias para se obter $160 \mathrm{~kg}$, os melhores resultados foram dos animais nascidos nos meses julho a setembro. A idade da vaca influenciou as caracteristicas ganho de peso pré-desmame e no D160.

Palavras chaves: ambiente e zebu.

ABSTRACT - The objective of this study was to evaluate the effects of environmental factors and age of dam on pre- (GPD) and post-weaning (GPS) daily gain, and on the were number of days to gain $160 \mathrm{~kg}$ (D160) from birth to weaning. The data set contained 1,747 animals, and were analyzed by the least squares method. The statistical model included the fixed effects of month and year of birth, and sex of the calf and age of the dam at calving. Sire nested within farm and the error were random effects. The pre- and post-weaning average daily gains, and days to gain $160 \mathrm{~kg}$ least squares means were $0.604 \pm 0.01 \mathrm{~kg}, 0.399 \pm 0.01 \mathrm{~kg}$, and $285.0 \pm 5.3$ days, respectively. The males were $6.0,21.1$ and $5.8 \%$ superior to the females for GPD, GPS and D160, respectively. The highest pre-weaning gain was for the animals born August. Regarding D160, the best results were for the animals born from July to September. Age of the cow showed a significant quadratic effect on the traits. The best cows were the 94-month-old ones. First calving cows produced the lightest calves. The results showed the importance of the environmental effects on the traits studied, evidencing the need for them to be corrected.

Key words: Environmental and Zebu.

\section{Introdução}

A pecuária de corte nacional, com mais de 185 milhões de cabeças (IBGE, 2003), na busca de maximizar o sistema de produção e se ajustar às mudanças políticas e sócio- econômicas que acontecem atualmente no planeta vem utilizando-se de novas tecnologias em seus programas de manejo e melhoramento animal (FERRAZ FILHO, 2001; MALHADO et al. , 2003). Nesse âmbito avaliar e aumentar os conhecimentos de 
características de desempenho produtivo e reprodutivo dos bovinos de corte, por regiões específicas é mais um diferencial que pode vir auxiliar os criadores a aumentar a produtividade do rebanho. Um país como o Brasil, com 8,5 milhões de $\mathrm{km}^{2}$, apresenta significativa diversidade de regiões, com diferentes sistemas de produção (ARRUDA e SUGAY, 1994) ocorrendo interações significativas dos efeitos de meio sobre a produção dos animais (SOUZA, 1997; EUCLIDES FILHO et al., 1998; SOUZA et al., 1998; SOUZA et al., 2004).

Identificar os animais mais precoces e a necessidade de se produzir animais à desmama mais elevados, permite que o mercado receba animais acabados com uma idade na faixa de 16 a 26 meses, dependendo do sistema de produção (EUCLIDES FILHO et al., 1998). Entretanto, essa busca pelo peso ótimo não depende somente do sistema de produção, mas também da relação custo/ benefício do investimento (KLOSTERMAN e PARKER, 1976; JENKINS e FERREL, 1983). Entre as diferentes metodologias usadas para se selecionar, FRIES et al. (1996) propuseram utilizar como critérios de seleção, dias para se atingir determinado peso, ao invés de se buscar o maior peso indefinidamente.

Segundo FERRAZ FILHO (2001) o desempenho econômico do sistema de produção é atribuído em função do ambiente, do genótipo e da interação entre estes, fazendo-se necessário à avaliação do binômio genótipo-ambiente (SOUZA, 1997; EUCLIDES FILHO et al., 1998). A influência de fatores ambientais tais como fazenda, mês e ano de nascimento, sexo e idade da vaca ao parto, relatados por MALHADO et al. (2002), BOCCHI et al. (2002) e por SOUZA et al. (2002) entre outros, realçam esses fatores, tornando-os importantes fontes de variação das características de produção em gado de corte.

Os objetivos deste trabalho foram avaliar os efeitos de meio e da idade da vaca sobre o ganho de peso do nascimento ao desmame, ganho de peso no pós-desmame e dias necessários para se obter $160 \mathrm{~kg}$ na fase pré-desmama.

\section{Material e Método}

As informações que integram o Sistema de Apoio ao Melhoramento do Zebu mantido pelo convênio ABCZ/EMBRAPA, foram cedidas pelo Centro Nacional de Pesquisa de Gado de Corte (CNPGC), unidade da EMBRAPA, Campo Grande (MS). O arquivo de dados continha 1.747 observações, de 194 touros distribuídos em 33 fazendas durante 20 anos, com animais nascendo durante os 12 meses, relativas a ganho de peso na fase de cria, recria e dias para se obter $160 \mathrm{~kg}$.

Os ganhos na fase de cria (GND) foram obtidos utilizando o peso ajustado aos 205 dias (P205) menos o peso ao nascimento dividido por 205. Para o ganho pós desmame, subtraiu-se o P205 do peso ajustado aos 550 dias (P550) e dividiu-se por 345. A característica dias para se atingir $160 \mathrm{~kg}$ de peso vivo (D160), foi obtida dividindo-se 160 $\mathrm{kg}$ pelo ganho de peso médio diário no período do nascimento ao desmame. Vale ainda salientar que dias para ganhar $160 \mathrm{~kg}$ é livre do peso ao nascer, ou seja a essa idade deve-se somar mais o peso ao nascer do animal.

O número de dias para ganhar $160 \mathrm{~kg}$ do nascimento ao desmame (D160) foi calculado com a seguinte fórmula:

$$
\mathrm{D} 160=\frac{160 \mathrm{~kg}}{\mathrm{GND}}
$$

Para D160 eliminou-se os animais com ganhos inferiores a $0,100 \mathrm{~kg}$ por serem considerados inaptos para seleção.

Os dados foram analisados pelo método dos quadrados mínimos utilizando-se o seguinte modelo estatístico:

$$
\begin{aligned}
& Y_{i j / m n o p}=\mu+T(F)_{i j}+M_{1}+A_{m}+S_{n}+b_{1}\left(X_{i j / m n o p}-\bar{X}\right)+b_{2} \\
& \left(X_{i j / m n o}^{2}-\bar{X}^{2}\right)+e_{i j / m n o p}
\end{aligned}
$$

em que:

$Y_{\text {iilmnop }}=$ variável dependente GPD, GPS, D160 do o-ésimo filho do touro $i$ dentro da; $\mu=$ média geral para as características analisadas; $T(F)_{i j}=$ efeito aleatório do reprodutor $i$ aninhado dentro da fazenda $j$; 
Efeito do meio e da idade da vaca sobre o ganho de pesa na fase de crias, recria e os dias para se obter...

$M_{1}=$ efeito fixo do mês $/$ de nascimento; $A_{m}$

$=$ efeito fixo do ano de nascimento $m ; S_{n}=$ efeito fixo do sexo da cria $n ; X_{i j / m n o}=$ efeito da o-esioma idade da vaca ao parto; $b_{1}=$ coeficiente de regressão para idade linear; $b_{2}=$ coeficiente de regressão para idade quadrática; e, $e_{i j \mid m n o}=$ erro aleatório, normal e independentemente distribuído com média zero e variância $o^{2}=1$.

\section{Resultados e Discussão}

O resumo da análise de variância dos dados (TABELA 1) mostra que várias das fontes de variações apresentaram efeito significativo $(P<0,05)$ sobre as características estudadas.

TABELA 1 - RESUMO DAS ANÁLISES DE VARIÂNCIA DE DIAS PARA SE OBTER 160KG (D160), GANHO DE PESO NA FASE DE CRIA (GPD) E GANHO DE PESO NO PÓS-DESMAME (GPS), EM VACAS DA RAÇA GUZERÁ ( $N=1747)$.

\begin{tabular}{lrrcc}
\hline Fontes de variação & \multicolumn{3}{c}{ GL } & \multicolumn{2}{c}{ Quadrados Médios $\left(\mathrm{kg}^{2}\right)$} \\
\hline & & D160 & GPD & GPS \\
NP( faz) & 259 & $9678,27^{* * *}$ & $0,0417^{* * *}$ & $0,0546^{* * *}$ \\
SEXO & 1 & $70603,46^{* * *}$ & $0,3807^{* * *}$ & $2,1651^{* * *}$ \\
MN & 11 & $13908,39^{* * *}$ & $0,0560^{* * *}$ & $0,0241 \mathrm{~ns}$ \\
AN & 19 & $4958,73^{*}$ & $0,0162^{*} \mathrm{~ns}$ & $0,0704 * *$ \\
IV (linear) & 1 & $19356,65^{*}$ & $0,0533^{*}$ & $0,0011 \mathrm{~ns}$ \\
IV (quadrático) & 1 & $25850,58^{* *}$ & $0,0745^{* *}$ & $0,0110 \mathrm{~ns}$ \\
\hline
\end{tabular}

$\mathrm{NP}(\mathrm{faz})=$ número do pai aninhado dentro de fazenda; $\mathrm{MN}=$ mês de nascimento; $\mathrm{AN}=$ ano de nascimento; IV= idade da vaca; ${ }^{* * *}=\mathrm{P}<0,0001 ;{ }^{* *}=\mathrm{P}<0,01 ;{ }^{*}=\mathrm{P}<0,05 ;$ ns $=$ não significativo.

As médias ajustadas pelo método dos quadrados mínimos para ganho de peso pré e pós-desmame, dias para ganhar $160 \mathrm{~kg}$ foram $0,604 \pm 0,01 \mathrm{~kg} ; 0,399 \pm$ $0,01 \mathrm{~kg} ; 285 \pm 5,3$ dias, respectivamente. Considerando-se que a característica D160 representa o $\mathrm{n}^{\circ} \mathrm{de}$ dias que o animal levou para ganhar 160 $\mathrm{kg}$ após o nascer, GARNEIRO et al. (2001) estudando animais da raça Nelore do programa de melhoramento da raça Nelore (PMGRN), encontraram a média de 245 dias. MALHADO (2003) trabalhando com animais da mesma raça, na região Nordeste do Brasil, verificou a média igual a 265,2 dias.

$O$ efeito de touro dentro de fazenda mostrou-se altamente significativo para as três características estudadas, confirmando os relatos de EUCLIDES FILHO et al. (1991) e SOUZA et al. (2004) entre outros, sobre a importância de se usar touros com diferencial positivo, os quais promovem o progresso genético do rebanho. $O$ uso de touros não avaliados e que podem ser portadores de
(DEPs) negativas em relação ao rebanho, trazem perdas como redução de precocidade e de ganho, não interessante para o criador. Para D160 dos 194 touros avaliados, observou-se média ajustada de $285 \pm 5,3$ dias, (média obtida dentro de um rol com um mínimo de 149 e um máximo de 476 dias). Para o ganho de peso até o desmame a média para touro dentro de fazenda foi 0,602 (média obtida dentro de um rol com um mínimo de 0,339 e o máximo de $1,022 \mathrm{~kg}$ ); e para o ganho de peso pós desmame a média foi de $0,407 \mathrm{~kg}$ (média obtida dentro de um rol com um mínimo de 0,154 e o máximo de 0,967 $\mathrm{kg})$.

O efeito de sexo como fonte de variação apresentou influência positiva $(P<0,001)$ sobre as características estudas. Os machos foram superiores às fêmeas para todas as características (TABELA 02), confirmando o dimorfismo sexual já relatado por outros autores como EUCLIDES FILHO et al. (1991), SOUZA et al. (2000), FERRAZ FILHO et al. (2002). 
TABELA 2 - PESOS AJUSTADOS PARAAS CARACTERÍSTICAS GANHOS PRÉ-DESMAME, GANHOS PÓS-DESMAME E DIAS PARA SE OBTER $160 \mathrm{KG}$, EM VACAS DA RAÇA GUZERÁ $(\mathrm{N}=1747)$.

\begin{tabular}{lccc}
\hline Sexo & GPD $(\mathrm{kg})$ & GPS $(\mathrm{kg})$ & D160 (dias) \\
\hline Macho & $0,622 \pm 0,01$ & $0,443 \pm 0,01$ & $277 \pm 5,6$ \\
Fêmea & $0,585 \pm 0,01$ & $0,354 \pm 0,01$ & $293 \pm 5,2$ \\
SM $^{1}(\%)$ & 6,0 & 20,1 & 5,8 \\
\hline
\end{tabular}

GPD = ganho pré-desmame; GPS = ganho pós-desmame; D160 = dias para ganhar $160 \mathrm{~kg}$.

O mês de nascimento como fonte de variação afetou tanto os ganhos de peso como dias para se ganhar $160 \mathrm{~kg}$. As maiores médias de ganho de peso pré-desmame foram observadas para os animais nascidos no mês de agosto, sendo que o intervalo de agosto a janeiro foi o que apresentou os maiores pesos. Isso confirma o fato de que as vacas que parem no final do período em que as pastagens estão escassas, entrando em meses de melhor oferta de pasto têm sua produção leiteira aumentada afetando positivamente o crescimento do bezerro nesse período. Estes bezerros, além de mais leite também encontram forragem de boa qualidade e em quantidade disponível, permitindo dando-lhes um melhor desempenho. Os maiores ganhos para GPS foram obtidos pelos animais nascidos no período compreendido entre dezembro e abril, com destaque para o mês de março, enquanto que os menores valores incidiram entre maio e dezembro. O mês de agosto apresentou situação atípica entre as médias, provavelmente devido a que os animais foram desmamados mais pesados e continuaram entre os mais pesados. Isso mostra uma relação entre qualidade e quantidade de pastagem com 0 desempenho dos animais.

GRÁFICO 1 - EFEITO DO MÊS DE NASCIMENTO DO BEZERRO SOBRE O GANHO DE PESO NA FASE DE CRIA (GPD) E NO PERÍODO PÓS DESMAME (GPS), NA RAÇA GUZERÁ.

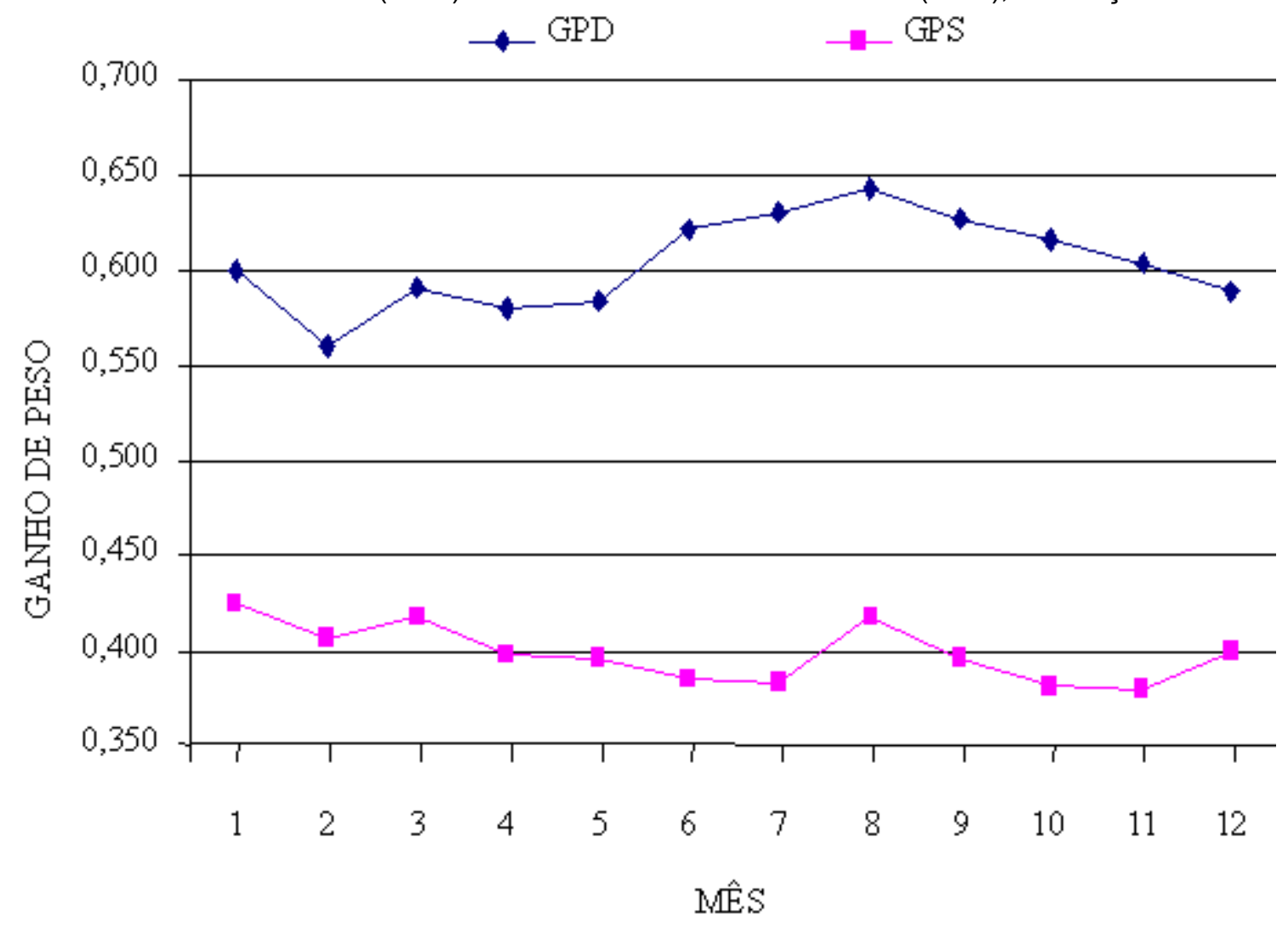


Efeito do meio e da idade da vaca sobre o ganho de pesa na fase de crias, recria e os dias para se obter...

Se o criador pretende comercializar animais ao desmame é importante que permaneça atento na escolha do melhor período para as vacas parirem. O uso de uma estação de monta pode auxiliar que os animais concentrem o nascimento em determinado período do ano trazendo vantagens. Por outro lado, ao se realizar recria ou terminação, a época de maior ganho altera-se, GRÁFICOS 1 e 2. Para se obter bons resultados (dias pra se ganhar $160 \mathrm{~kg}$ ) os animais devem ser gerados do mês de agosto, e não no de fevereiro em função de mais dias para se atingir $160 \mathrm{~kg}$.

GRÁFICO 2 - EFEITO DO MÊS DE NASCIMENTO SOBRE OS DIAS PARA OS GANHOS DE 160KG, NA RAÇA GUZERÁ.

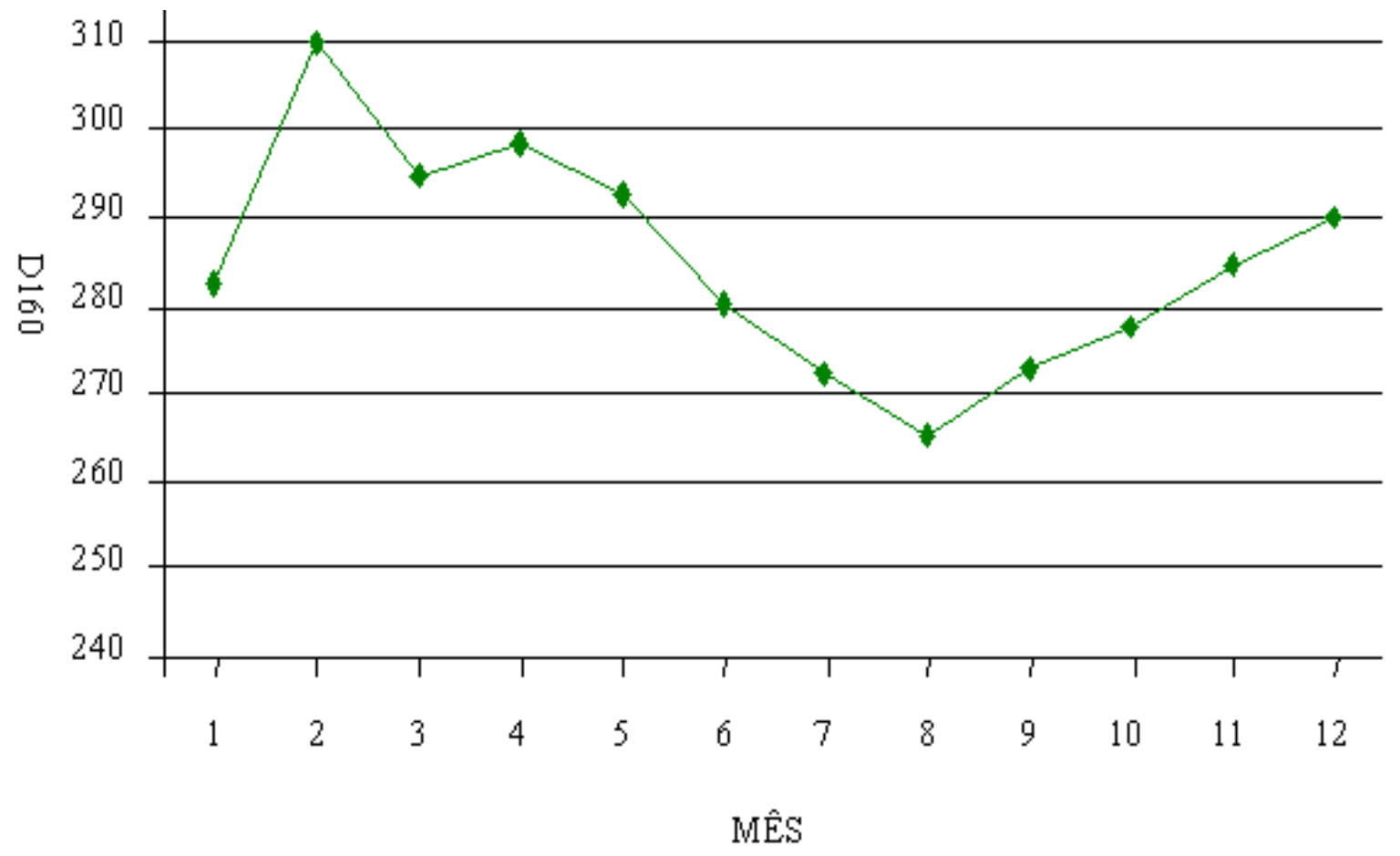

O ano de nascimento influenciou significativamente algumas das características estudadas, sendo que no período de 1975 à 1996, o menor ganho foi observado em 1980 quando os animais convertiam 0,295 $\pm 0,031$ $\mathrm{g} /$ dia e o melhor ganho aconteceu em 1985, $0,468 \pm 0,021 \mathrm{~g} / \mathrm{dia}$.

$O$ efeito da idade da vaca mostrou-se significativo $(P<0,05)$ nas características que envolvem a fase de cria. Essa teve sua fase ótima, quando suas progênies desmamaram mais pesadas aos 95 meses de idade e 93 meses, respectivamente para D160 e GPD, demostrando que a partir desse ponto o peso das progênies começam a cair, indicando que a matriz já pode ser descartada, GRÁFICOS 3 e 4.

\section{Conclusão}

Constatou-se o efeito significativo de touro dentro de fazenda, evidenciando mais uma vez a importância de se usar touros com diferencial conhecido e positivo. A implantação de estação de monta, visando otimizar o período de nascimentos, pode tornar-se vantajosa para quem comercializa bezerros ao desmame. A idade da vaca influenciou as caracteristicas avaliadas na fase de cria, sendo que as primíparas e aquelas com idade superior a 196 meses, geraram bezerros mais leves, devendo-se descartar às de idade mais avançada. 
GRÁFICOS 3 - EFEITO DA IDADE DA VACA (IV) EM MESES, SOBRE O GANHO DE PESO (GPD) NA FASE DE CRIA.

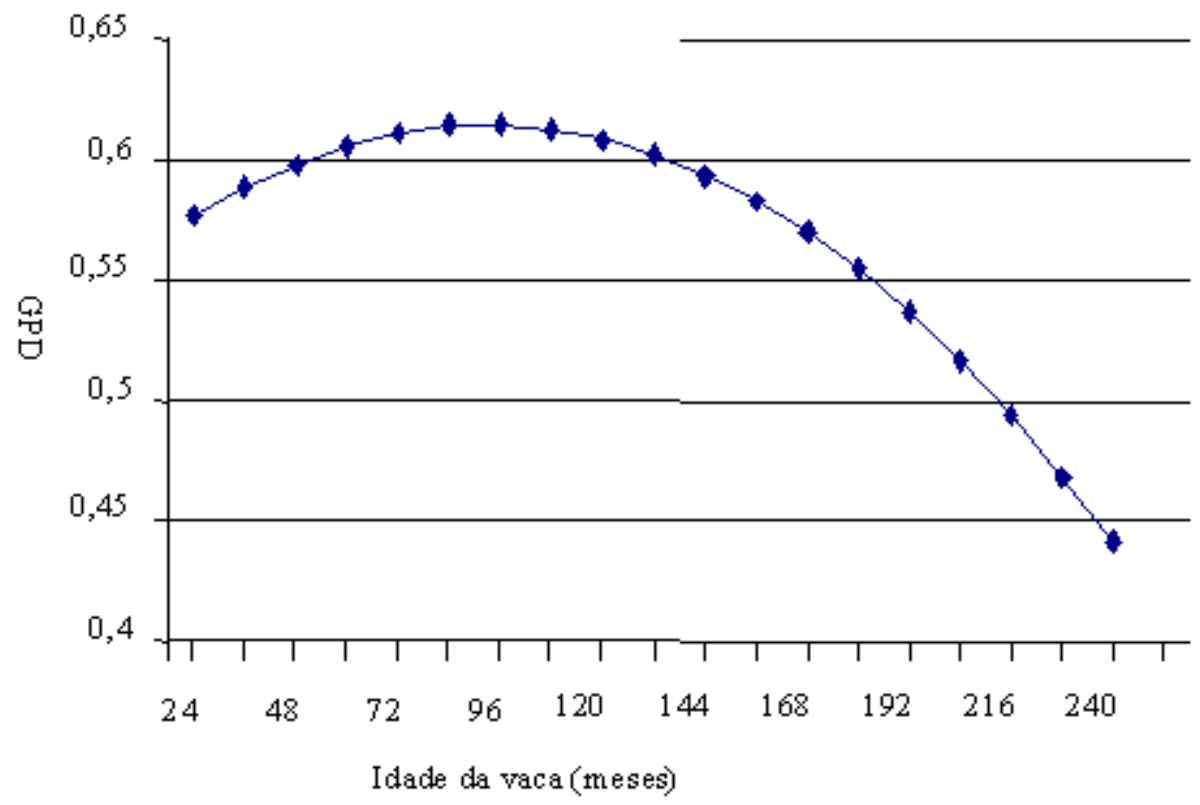

GRÁFICO 4 - EFEITO DA IDADE DA VACA E OS DIAS PARA OS GANHOS DE 160 KG (D160).

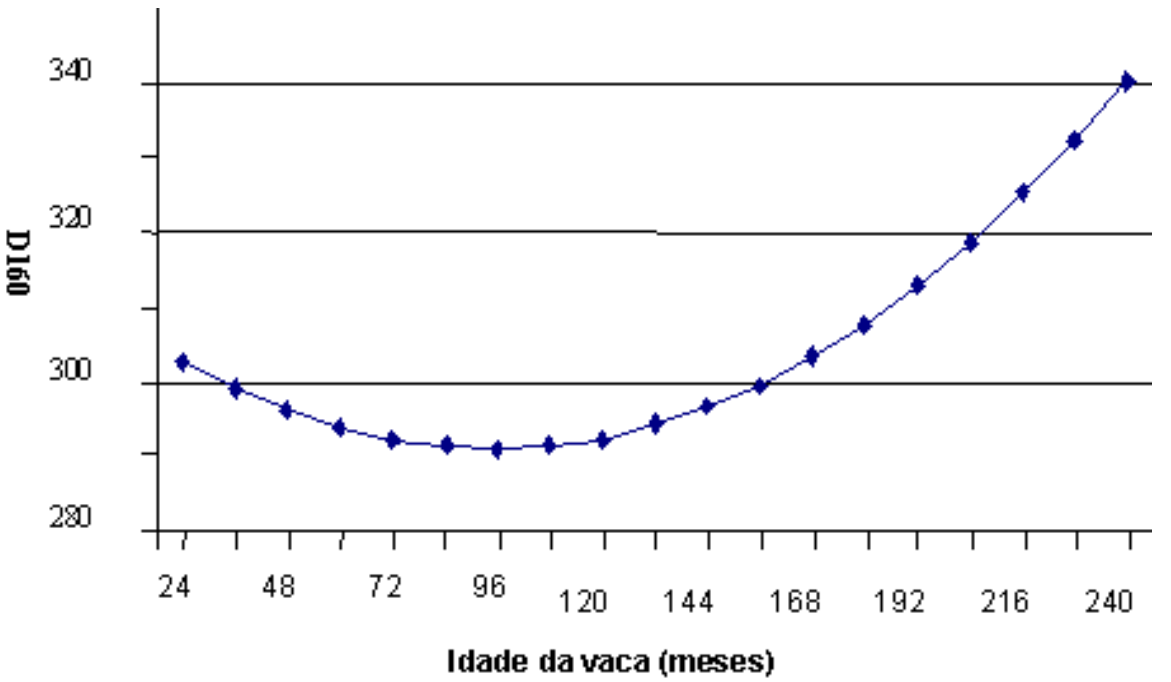

\section{Referências}

ARRUDA, Z.J.; SUGAY 1994. Regionalização da pecuária bovina no Brasil. Campo Grande: EMBRAPA-CNPGC; Brasília: EMBRAPA-SPI. $144 p$ - (EMBRAPA-CNPGC, documentos, 58).

BOCCHI, A.L.; TEIXEIRA, R.; ALBUQUERQUE, L.G. Efeito da idade da vaca ao parto sobre o peso ao desmame na raça nelore em diferentes estados brasileiros. In: XXXIX Reunião Anual da Sociedade Brasileira de Zootecnia. 2002. Recife-PE. Anais. Recife: Sociedade Brasileira de Zootecnia, 2002. CD-Rom.
EUCLIDES FILHO, K.; NOBRE, P.R.C.; ROSA, A.N. Idade da vaca e suas interrelações com a fazenda, reprodutor $e$ sexo do bezerro. Revista Brasileira de Zootecnia, Viçosa, v.20, p.40-6. 1991.

EUCLIDES FILHO, K.; FIGUEIREDO, G.R.; SILVA, L.O.C.; ALVES, R.G.O . Idade aos $165 \mathrm{~kg}$ de peso vivo para progênies de Nelore, Fleckvieh, Chianina, Charolês, F1's e Retrocruzas. Revista Brasileira de Zootecnia, Viçosa, v.27, p.899-905, 1998. 
Efeito do meio e da idade da vaca sobre o ganho de pesa na fase de crias, recria e os dias para se obter...

FERRAZ FILHO, P.B. Avaliação genética do desenvolvimento ponderal de bovinos da raça Tabapuã no Brasil. Botucatu, 2001, 135p. Tese (Doutorado em Genética) Instituto de Biociências, Universidade Estadual Paulista, 2001.

FERRAZ FILHO, P.B.; RAMOS, A.A.; SILVA, L.O.C.; SOUZA, J.C.; ALENCAR, M.M. Herdabilidades e correlações genéticas, fenotípicas e ambientais para pesos em diferentes idades de bovinos da raça Tabapuã. Archives of Veterinary Science, Curitiba, v.7, n.1, p.75-79, 2002.

FRIES, L.A.; BRITO, F.V.; ALBUQUERQUE, L.G. Possíveis conseqüências de seleção para incrementar pesos a idades - padrão vs reduzir idade para produzir unidade de mercado. In: REUNIÃO ANUAL DA SOCIEDADE BRASILEIRA DE ZOOTECNIA, 33, 1996, Fortaleza, CE. Anais... Fortaleza : SBZ. p.310-312. 1996.

GARNERO, A.D.V.; LOBO, R.B.; BEZERRA, L.A.F.; OLIVEIRA, H.N. Comparação entre Alguns Critérios de Seleção para Crescimento na Raça Nelore. Revista Brasileira de Zootecnia, Viçosa, v.30, n.3, p.714-718, 2001.

IBGE - Efetivo dos rebanhos - Brasil. 2003. www.ibge.gov.br. Consultado em novembro de 2003.

JENKINS, T.G.; FERREL, C.L. Estimated production efficiencies of crossbred cows. Journal of Animal Science, Savoy, v.57, suppl.1, p.154, 1983.

KLOSTERMAN, E.W.; PACKER, C.F. Effect of size, breed and sex upon feed efficiency in beef cattle. Ohio agricultural Research and development Center Research Bulletin, Wooster, 1088, 39p., 1976.

MALHADO, C.M.M. Avaliação de critérios de seleção relacionados a velocidade de crescimento em bovinos da raça Nelore no Nordeste do Brasil. Fortaleza,45p. Dissertação (Mestrado em Zootecnia - Produção Animal). Universidade Federal do Ceará, 2003.
MALHADO, C.M.M.; MARTINS FILHO, R.; LÔBO, R.N.B.; AZEVÊDO, D.M.M.R. Parâmetros genéticos para características relacionadas à velocidadede crescimento em bovinos nelore. In: Congresso Brasileiro das Raças Zebuinas, 5, 2002, Uberaba,MG. Anais... Uberava:ABCZ. p271, 2002.

SOUZA, J.C. Interação genótipo $x$ ambiente sobre o peso ao desmame de zebuínos da raça Nelore no Brasil. Botucatu, 122p. Tese (Doutorado em Genética). Universidade Estadual Paulista, 1997.

SOUZA, J.C.; MALHADO, C.H.M.; SILVA, L.O.C.; PIRES, A.G.; BALDO, F.; PIEKARSKI, P.R.B.; FERRAZ FILHO, P.B. Causas de variação e tendência genética para dias para ganhar 160 e $240 \mathrm{~kg}$ em bovinos Guzerá criados na região nordeste do Brasil. In: SIMPÓSIO NACIONAL DE MELHORAMENTO ANIMAL, 4, 2002, Campo Grande, MS. Anais... IV Simpósio Nacional de Melhoramento Animal, v.4, p.204-207, 2002.

SOUZA, J.C.; RAMOS, A.A.; SILVA, L.O.C.; EUCLIDES FILHO, K.; ALENCAR, M.M.; WECHSLER, F.S.; GADINI, C.H.; VAN VLECK, L.D. Effect of genotype $x$ enviroment interaction on weaning weight of Nellore calves raised in four different regions of Brazil. In: WORLD CONGRESS OF GENETICS APPLIED TO LIVESTOCK PRODUCTION, 6, 1998, Armidale, Australia. Anais... $6^{\text {Th }}$ World Congress on Genetics Applied to Livestock Production. v.23, p.196-198, 1998.

SOUZA, J.C.; SILVA, L.O.C.; RAMOS, A.A.; FERRAZ FILHO, P.B.; CÍCERO, E.A.; MALHADO, C.H.M. Fatores ambientais, genéticos e interação touro $x$ fazenda sobre o peso aos 205 dias de idade em bovinos de raça Nelore na região de Campo Grande Dourados, MS. Trabalho no prelo dos Arquivos de Ciências Veterinárias e Zoologia da UNIPAR, 2004.
Recebido para publicação: 14/01/2004

Aprovado:
$15 / 05 / 2004$ 